Journal of Mathematics and Statistics 3 (3): 106-108, 2007

ISSN 1549-3644

(C) 2007 Science Publications

\title{
Bayes Estimation of the Lifetime Parameters for the Exponential Distribution
}

\author{
Ahmed A. Abu-Taleb, Mahmoud M. Smadi and Ameen J. Alawneh \\ Department of Mathematics and Statistics \\ Jordan University of Science and Technology \\ P.O. BOX 3030 Irbid, 22110, Jordan
}

\begin{abstract}
Bayes estimation for the parameters of the lifetime distribution when both survival and censoring time are exponentially distributed were studied. The marginal posteriors, Bayes estimates and credible sets were derived using conjugate priors.
\end{abstract}

Keywords: Bayes estimation; credible sets, survival functions

\section{INTRODUCTION}

There are many scenarios in life-testing and reliability experiments in which units are lost or removed from the test before failure. The Bayesian approach requires specifying a probability distribution for the observed data and a prior probability of possible distributions, then all the inference is based on the posterior distribution given the data. Many authors have discussed the Bayesian approach for different scenarios or distributions in both parametric and nonparametric survival data. A Bayes method to infer an unknown failure time distribution method based on the piecewise exponential distribution and a relationship between values of the failure rate in successive intervals presented by Gamerman ${ }^{[3]}$; it provides smooth estimates of the survival and hazard functions of the distribution. Bayesian survival estimation of the Pareto distribution of the second kind on failure-censored data was proposed by Howlader and Hossian ${ }^{[4]}$, Mostert et al. ${ }^{[5]}$ presented the Bayesian analysis of survival data using the Rayleigh model and linex loss, Mostert et al. [6] introduced the Bayes estimators of the lifetime parameters using the compound Rayleigh model. A nonparametric Bayesian estimation of survival curves from incomplete observations was discussed by Susarla and Van Ryzin [7]. In this paper, a parametric Bayes estimation for the lifetime parameters were discussed when the lifetime and the censoring time are exponentially distributed.
A randomly censored data set consists of $n$ i.i.d pairs $\left(Y_{i}, D_{i}\right)$, where $Y_{i}=\operatorname{Min}\left(X_{i}, T_{i}\right)$ and $D_{i}=I\left(X_{i}\right.$ $\left.\leq T_{i}\right)$ for $i=1,2, \ldots n$. In the context of survival analysis and reliability, $X_{i}$ refers to the survival time and $T_{i}$ refers to the censoring time. Assume that $X_{i}$, the survival time, $i=1,2, \ldots n$, and $T_{i}$, censoring time, are independent exponentially distributed with probability density functions given by:

$$
\mathrm{f}_{\mathrm{X}_{\mathrm{i}}}\left(\mathrm{x}_{\mathrm{i}}, \theta\right)=\frac{1}{\theta} \mathrm{e}^{-\mathrm{x}_{\mathrm{i}} / \theta}, \quad \mathrm{x}_{\mathrm{i}}>0, \theta>0
$$

and

$\mathrm{f}_{\mathrm{T}_{\mathrm{i}}}\left(\mathrm{t}_{\mathrm{i}}, \lambda\right)=\frac{1}{\lambda} \mathrm{e}^{-\mathrm{t}_{\mathrm{i}} / \lambda}, \quad \mathrm{t}_{\mathrm{i}}>0, \lambda>0$

respectively. Suppose we observe $\left[\left(Y_{i}, D_{i}\right) ; i=1,2, n\right]$, then the probability density function (p.d.f) of $D_{i}$ is:

$$
\begin{aligned}
\mathrm{P}\left(\mathrm{D}_{\mathrm{i}}=1\right) & =\mathrm{P}\left(\mathrm{X}_{\mathrm{i}} \leq \mathrm{T}_{\mathrm{i}}\right) \\
& =\int_{0}^{\infty} \int_{\mathrm{x}_{\mathrm{i}}}^{\infty} \frac{1}{\theta} \mathrm{e}^{-\mathrm{x}_{\mathrm{i}} / \theta} \frac{1}{\lambda} \mathrm{e}^{-\mathrm{t}_{\mathrm{i}} / \lambda} \mathrm{dt}_{\mathrm{i}} \mathrm{dx}_{\mathrm{i}} \\
& =\frac{\lambda}{\lambda+\theta}
\end{aligned}
$$

and hence,

$\mathrm{P}\left(\mathrm{D}_{\mathrm{i}}=0\right)=\frac{\theta}{\lambda+\theta}$.

It can be shown that the joint probability density function of $\left(\mathrm{Y}_{\mathrm{i}}, \mathrm{D}_{\mathrm{i}}\right)$ is:

Corresponding Author: Ahmed A. Abu-Taleb, Department of Mathematics and Statistics, Jordan University of Science and Technology, P. O. Box 3030, Irbid, 22110, JORDAN 
$\mathrm{f}_{\mathrm{Y}_{\mathrm{i}}, \mathrm{D}_{1}}\left(\mathrm{y}_{\mathrm{i}}, \mathrm{d}_{\mathrm{i}}, \theta, \lambda\right)=\left(\frac{1}{\theta}+\frac{1}{\lambda}\right)^{-\left(\frac{1}{\theta}+\frac{1}{\lambda}\right) \mathrm{y}_{\mathrm{i}}}\left(\frac{\lambda}{\lambda+\theta}\right)^{\mathrm{d}_{\mathrm{i}}}\left(\frac{\theta}{\lambda+\theta}\right)^{1-\mathrm{d}_{\mathrm{i}}}, \mathrm{y}_{\mathrm{i}}>0, \theta, \lambda>0$

and the likelihood function is given by

$$
L(\theta, \lambda)=\left(\frac{1}{\theta}+\frac{1}{\lambda}\right)^{\mathrm{n}} \mathrm{e}^{-\left(\frac{1}{\theta}+\frac{1}{\lambda}\right) \sum_{i=1}^{\mathrm{n}} \mathrm{y}_{\mathrm{i}}}\left(\frac{\lambda}{\theta}\right)^{\sum_{i=1}^{\mathrm{n}} \mathrm{d}_{\mathrm{i}}}\left(\frac{\theta}{\lambda+\theta}\right)^{\mathrm{n}}
$$

It is shown that the maximum likelihood estimators of $\theta$ and $\lambda$ are given by (See Abu-Taleb and Smadi, ${ }^{[1]}$ )

$$
\hat{\theta}=\frac{\sum_{i=1}^{n} Y_{i}}{\sum_{i=1}^{n} D_{i}}
$$$$
\hat{\lambda}=\frac{\sum_{i=1}^{n} Y_{i}}{n-\sum_{i=1}^{n} D_{i}}
$$

\section{BAYES ESTIMATION}

We consider the following informative priors on the parameters $\theta$ and $\lambda$

$$
\begin{aligned}
\pi(\theta, \lambda) & =\pi_{1}(\theta) \pi_{2}(\lambda) \\
& =\frac{\mathrm{b}_{1}^{\mathrm{a}_{1}}\left(\frac{1}{\theta}\right)^{\mathrm{a}_{1}-1} \mathrm{e}^{-\frac{\mathrm{b}_{1}}{\theta}}}{\Gamma\left(\mathrm{b}_{1}\right)} \frac{\mathrm{a}_{2}\left(\frac{1}{\lambda}\right)^{\mathrm{a}_{2}-1} \mathrm{e}^{-\frac{\mathrm{b}_{2}}{\lambda}}}{\Gamma\left(\mathrm{a}_{2}\right)}, \quad \theta>0, \lambda>0 .
\end{aligned}
$$

This prior assumes the independence of $\theta$ and $\lambda$ with an inverted gamma prior on $\theta$ with hyper parameters $a_{l}$ and $b_{1}$, and an inverted gamma prior on $\lambda$ with hyper parameters $a_{2}$ and $b_{2}$.

The likelihood function of $\theta$ and $\lambda$ for fixed $\mathbf{X}$ and $\mathbf{D}$ is given by

$$
\pi(\mathrm{X}, \mathrm{D} \mid \theta, \lambda)=\left(\frac{1}{\theta}+\frac{1}{\lambda}\right)^{\mathrm{n}} \mathrm{e}^{-\left(\frac{1}{\theta}+\frac{1}{\lambda}\right) \sum_{i=1}^{\mathrm{n}} \mathrm{y}_{\mathrm{i}}}\left(\frac{\lambda}{\theta}\right)^{\sum_{i=1}^{\mathrm{n}} \mathrm{d}_{\mathrm{i}}}\left(\frac{\theta}{\lambda+\theta}\right)^{\mathrm{n}}
$$

Using Bayes theorem, the joint posterior distribution is given by

$$
\pi(\theta \lambda \mid \quad)=\frac{\pi(\theta \lambda) \pi(\quad \mid \theta \lambda)}{\int \pi(\theta \lambda) \pi(\quad \mid \theta \lambda) d \theta d \lambda}
$$

or

$$
\pi(\theta, \lambda \mid \mathrm{X}, \mathrm{D}) \propto \pi(\theta, \lambda) \pi(\mathrm{X}, \mathrm{D} \mid \theta, \lambda)
$$

Combining the likelihood function given in (4) with the joint prior distribution given in (3) yields:

$$
\begin{aligned}
\pi(\theta, \lambda \mid X, D) \propto \frac{b_{1}^{a_{1}}\left(\frac{1}{\theta}\right)^{a_{1}-1} e^{-\frac{b_{1}}{\theta}}}{\Gamma\left(a_{1}\right)} \frac{b_{2}^{a_{2}}\left(\frac{1}{\lambda}\right)^{a_{2}-1} e^{-\frac{b_{2}}{\lambda}}}{\Gamma\left(a_{2}\right)} x \\
\left(\frac{1}{\theta}+\frac{1}{\lambda}\right)^{n} e^{-\left(\frac{1}{\theta}+\frac{1}{\lambda} \sum_{i=1}^{n} y_{i}\left(\frac{\lambda}{\theta}\right)^{\sum_{i=1}^{n} d_{i}}\left(\frac{\theta}{\lambda+\theta}\right)^{n}\right.}
\end{aligned}
$$

after simplification we get

$$
\begin{gathered}
\pi(\theta, \lambda \mid \mathrm{y}, \mathrm{d}) \propto\left(\frac{1}{\theta}\right)^{\sum_{i=1}^{\mathrm{n}} \mathrm{d}_{\mathrm{i}}+\mathrm{a}_{1}-1} \mathrm{e}^{-\frac{1}{\theta}\left(\mathrm{b}_{1}+\sum_{\mathrm{i}=1}^{\mathrm{n}} \mathrm{y}_{\mathrm{i}}\right)} \mathrm{x} \\
\left(\frac{1}{\lambda}\right)^{\mathrm{n}-\sum_{\mathrm{i}=1}^{\mathrm{n}} \mathrm{d}_{\mathrm{i}}+\mathrm{a}_{2}-1} \mathrm{e}^{-\frac{1}{\lambda}\left(\mathrm{b}_{2}+\sum_{\mathrm{i}=1}^{\mathrm{n}} \mathrm{y}_{\mathrm{i}}\right)}
\end{gathered}
$$

The factors involving $\theta$ and $\lambda$ in this last expression are clearly recognizable as inverted gamma posterior distributions. The marginal posterior of $\theta$ follows an inverted gamma distribution with parameters $\sum_{\mathrm{i}=1}^{\mathrm{n}} \mathrm{d}_{\mathrm{i}}+\mathrm{a}_{1}$ and $\sum_{\mathrm{i}=1}^{\mathrm{n}} \mathrm{y}_{\mathrm{i}}+\mathrm{b}_{1}$, also, the marginal posterior of $\lambda$ follows an inverted gamma distribution with parameters $n-\sum_{i=1}^{n} d_{i}+a_{2}$ and $\sum_{i=1}^{n} y_{i}+b_{2}$; hence the Bayes estimator of $\theta$ with respect to squared-error loss function is the mean of the marginal posterior distribution of $\theta$ given $\sum_{i=1}^{n} d_{i}$ and $\sum_{i=1}^{n} y_{i}$ and is given by

$\hat{\theta}_{B}=\frac{\sum_{i=1}^{n} Y_{i}+b_{1}}{\sum_{i=1}^{n} D_{i}+a_{1}}$

Similarly, the Bayes estimator of $\lambda$ is

$$
\hat{\lambda}_{B}=\frac{\sum_{i=1}^{n} Y_{i}+b_{2}}{n-\sum_{i=1}^{n} D_{i}+a_{2}}
$$

As expected, the Bayes estimators updated the information in the observed sample with the information using the prior information specified in the hyper parameters. 
To derive the credible sets, if $\pi(\theta \mid x)$ is the posterior distribution of $\theta$ given $\mathbf{X}=\mathbf{x}$, then for any set $A \subset \Theta$, the credible probability of $A$ is

$\mathrm{P}(\theta \in \mathrm{A} \mid \mathrm{x})=\int_{\mathrm{A}} \pi(\theta \mid \mathrm{x}) \mathrm{d} \theta$

and $A$ is a credible set for $\theta$.

Given that the marginal posterior of $\theta$ follows an inverted gamma distribution with parameters $\sum_{\mathrm{i}=1}^{\mathrm{n}} \mathrm{d}_{\mathrm{i}}+\mathrm{a}_{1}$ and $\sum_{\mathrm{i}=1}^{\mathrm{n}} \mathrm{y}_{\mathrm{i}}+\mathrm{b}_{1}$, we can form a credible set for $\theta$ in many different ways, as any set $A$ satisfying (9) will do. One simple way is to split the $\alpha$ equally between the upper and lower endpoints. It follows that $2\left(\sum_{\mathrm{i}=1}^{\mathrm{n}} \mathrm{y}_{\mathrm{i}}+\mathrm{b}_{1}\right) \theta \sim \chi_{2\left(\sum_{\mathrm{i}=1}^{\mathrm{n}} \mathrm{d}_{\mathrm{i}}+\mathrm{a}_{1}\right)}$

Thus a $1-\alpha$ credible interval is

$$
\left\{\theta: \frac{2\left(\sum_{i=1}^{n} y_{i}+b_{1}\right)}{\chi^{2}} \sum_{2\left(n-\sum_{i=1}^{n} d_{i}+a_{1}\right), 1-\frac{\alpha}{2}}^{n} \leq \theta \leq \frac{2\left(\sum_{i=1}^{n} y_{i}+b_{1}\right)}{\chi^{2}\left(n-\sum_{i=1}^{n} d_{i}+a_{1}\right), \frac{\alpha}{2}}\right\} .
$$

If $a_{1}=b_{1}=0$, the posterior distribution of $\theta$ given $\sum_{\mathrm{i}=1}^{\mathrm{n}} \mathrm{d}_{\mathrm{i}}$ and $\sum_{\mathrm{i}=1}^{\mathrm{n}} \mathrm{y}_{\mathrm{i}}$ can then be expressed as $2\left(\sum_{\mathrm{i}=1}^{\mathrm{n}} \mathrm{y}_{\mathrm{i}}\right) \theta \sim \chi_{2\left(\sum_{\mathrm{i}=1}^{2} \mathrm{~d}_{\mathrm{i}}\right)}^{\mathrm{n}}$.

Given that the marginal posterior of $\lambda$ follows an inverted gamma distribution with parameters $\mathrm{n}-\sum_{\mathrm{i}=1}^{\mathrm{n}} \mathrm{d}_{\mathrm{i}}+\mathrm{a}_{2}$ and $\sum_{\mathrm{i}=1}^{\mathrm{n}} \mathrm{y}_{\mathrm{i}}+\mathrm{b}_{2}$, also it follows that (See Casella and Berger, ${ }^{[2]}$ )

$$
2\left(\sum_{\mathrm{i}=1}^{\mathrm{n}} \mathrm{y}_{\mathrm{i}}+\mathrm{b}_{2}\right) \lambda \sim \chi_{2\left(\mathrm{n}-\sum_{\mathrm{i}=1}^{\mathrm{n}} \mathrm{d}_{\mathrm{i}}+\mathrm{a}_{2}\right)}
$$

Thus a $1-\alpha$ credible interval is

$$
\left\{\lambda: \frac{2\left(\sum_{i=1}^{n} y_{i}+b_{2}\right)}{\chi_{2\left(n-\sum_{i=1}^{2} d_{i}+a_{2}\right), 1-\frac{\alpha}{2}}^{2}} \leq \lambda \leq \frac{2\left(\sum_{i=1}^{n} y_{i}+b_{2}\right)}{\chi_{2\left(n-\sum_{i=1}^{2} d_{i}+a_{2}\right), \frac{\alpha}{2}}^{2}}\right\} .
$$

If $a_{2}=b_{2}=0$, the posterior distribution of $\theta$ given $\sum_{i=1}^{n} d_{i}$ can then be expressed as

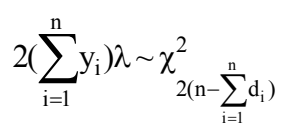

\section{CONCLUSION}

Bayes estimation for the parameters of the survival functions when both survival and censoring time are exponentially distributed were studied. The marginal posteriors, Bayes estimates and credible sets were derived using conjugate inverted gamma priors.

\section{REFERENCES}

1. Abu-Taleb A. Ahmed, Smadi, M. Mahmoud, 2006. Asymptotic Efficiencies of the Survival Functions Estimators for the Exponential Distribution. International Mathematical Forum, 1 (38): 18611869.

2. Casella G. and Berger R. L., 2002. Statistical Inference. Duxbury Press.

3. Gamerman, D., 1994. Bayes Estimation of the Piece-Wise Exponential Distribution, IEEE Transactions on Reliability, 43 (1): 128 - 131

4. Howlader, Hatem A. and Hossain, Anwar M., 2002. Bayesian Survival Estimation of Pareto Distribution of the Second Kind Based on FailureCensored Data. J. Comput. Stat. Data Anal., 38 (3): 301-314.

5. Mostert, P. J., Bekker, A. and Roux, J.J.J., 1998. Bayesian Analysis of Survival Data Using the Rayleigh Model and Linex Loss. South Africa Statistical Journal, 32 (1): 19-42.

6. Mostert, P. J., Roux, J.J.J. and. Bekker, A., 1999. Bayes Estimators of the Lifetime Parameters Using the Compound Rayleigh Model. South Africa Statistical Journal, 33 (2): 117-138.

7. Susarla and Van Ryzin, 1976. A Nonparametric Bayesian Estimation of Survival Curves from Incomplete Observations. Journal of American Statistical Association. 71: 897-902. 